\title{
COMPORTAMIENTO DE LA PAPA CRIOLLA (Solanum phureja Just et Buk) A CALCIO Y BORO EN UN SUELO DEL DEPARTAMENTO DE CUNDINAMARCA, COLOMBIA
}

\section{CREOLE POTATO (Solanum phureja Just et Buk) BEHAVIOR TO CALCIUM ANDA BORON IN A SOIL OF CUNDINAMARCA, COLOMBIA}

Andrés Segura ${ }^{1}$

Alfonso Triviño ${ }^{1}$

Rodrigo Lora Silva ${ }^{2}$

\section{RESUMEN}

En el municipio de Funza, Cundinamarca, en un suelo derivado de cenizas volcánicas clasificado como Typic Dystrandept, se evaluó en papa criolla (Solanum phureja Just et Buk) el efecto de la aplicación edáfica de calcio y de boro y su interacción en términos de rendimiento y de calidad de los tubérculos. Se utilizó un diseño experimental de bloques completos al azar en arreglo factorial completo, dos factores y cuatro niveles para un total de 48 unidades experimentales de $20 \mathrm{~m}^{2}$. Los niveles de calcio fueron $0-18-36-54 \mathrm{~kg} / \mathrm{ha}$ y de boro $0-0,5-1$ $1,5 \mathrm{~kg} \mathrm{~B} / \mathrm{ha}$. Se evaluó el rendimiento total y por tamaño del tubérculo, la humedad y la densidad y el análisis económico. Los resultados mostraron efecto significativo de los tratamientos en el rendimiento, obteniéndose los mayores con la aplicación de 18 y $54 \mathrm{~kg}-\mathrm{Ca} / \mathrm{ha}$, y de $1 \mathrm{~kg} \mathrm{~B} / \mathrm{ha}$ junto con $18 \mathrm{~kg}-\mathrm{Ca} / \mathrm{ha}$, con rendimiento total de 29,7; 30,2 y 22,5 t/ha, respectivamente. No hubo efecto en el tamaño de tubérculos, pero la mayoría de ellos fue de tamaño primera. Los mayores ingresos marginales, se obtuvieron con la aplicación de calcio solo, $1 \mathrm{~kg}$ de B y 18 de Ca y $1 \mathrm{~kg}$ de B y 36 de

\footnotetext{
${ }^{1}$ Ingenieros Agrónomos

${ }^{2}$ Ing. Químico, M.Sc., docente investigador, Facultad de Ingeniería Agronómica, Universidad de Ciencias Aplicadas y Ambientales U.D.C.A. Correo electrónico: rodrigolora@yahoo. com, Dirección para correspondencia: Calle 99 No. 48D-15, Unidad 5, Apto 2, Bogotá, D.C.
}

Ca. Por su parte, los niveles de 0,5; 1,0 y $1,5 \mathrm{~kg} \mathrm{~B} / \mathrm{ha}$ sin Ca produjeron ingresos marginales de \$253.400, $\$ 502.000$ y $\$ 885.000$, respectivamente. Aunque no hubo incremento significativo en la densidad de los tubérculos, se presentó incremento en relación al testigo para las combinaciones de $0,5 \mathrm{~kg} \mathrm{~B} / \mathrm{ha}$ y los niveles de $\mathrm{Ca}$, indicando la necesidad de la presencia del Boro con el Calcio.

Palabras clave: Fertilización, micro y nutrientes secundarios, tamaño de tubérculos, rentabilidad.

\section{SUMMARY}

At Funza, Cundinamarca, Colombia, in a soil derived from volcanic ashes, classified as Typic Dystrandept, the effect of Calcium and Boron applications and its interactions on yield and tuber quality of the creole potato was evaluated. A complete randomized block design with factorial arrangements, two factors and fore levels was used for a total of 48 experimental units of $20 \mathrm{~m}^{2}$ each. The levels of Ca were $0-18-36-54 \mathrm{~kg} / \mathrm{ha}$ and those of B 0 , $05,1,1.5 \mathrm{~kg} / \mathrm{ha}$. Total yield and tuber size, as well as tuber density and humidity and the economic analysis were evaluated. The results showed a significant effect of the treatments on the total yield, being the highest, obtained with 18 and $54 \mathrm{~kg} \mathrm{Ca} / \mathrm{ha}$ and $1 \mathrm{~kg} \mathrm{~B} / \mathrm{ha}$ with $18 \mathrm{~kg} \mathrm{Ca} / \mathrm{ha}$, 29.7, 30.2 y 22.5 t/ha, respectively. No effect on tuber size was observed, but production was mainly of the first class size. The highest marginal incomes were obtained with 
calcium alone, $1 \mathrm{~kg}$ of $\mathrm{B}$ and $18 \mathrm{~kg}$ of $\mathrm{Ca}$, and $1 \mathrm{~kg}$ of $\mathrm{B}$ and 36 of $\mathrm{Ca}$. The boron levels, $0.5,1.0$ and 1.5 without $\mathrm{Ca}$ produced marginal incomes of $\$ 253.400, \$ 502.000$ and $\$ 885.000$, respectively. Although no significant increase of the tuber density was obtained, in comparison with the check, for the combinations of $0.5 \mathrm{~kg} / \mathrm{ha}$ of $\mathrm{B}$ and the Ca levels, an increase was observed, indicating the necessity of the presence of B with Ca.

Key words: Fertilization, micro and secondary nutrients, tuber size, rentability

\section{INTRODUCCIÓN}

La papa criolla (Solanum phureja Just et Buk), se siembra en Colombia en una extensión aproximada de 12.000 hectáreas semestrales en los departamentos de Cundinamarca, Boyacá, Santander, Norte de Santander, Nariño y Antioquia, principalmente (Lora et al. 2006). Por sus propiedades culinarias y nutricionales, el tubérculo es de gran aceptación en la población colombiana y además tiene un alto potencial de exportación hacia Estados Unidos, Canadá, Japón y algunos países europeos.

En el país, la papa criolla se cultiva bajo condiciones similares a la dadas para la especie Solanum tuberosum, en la cual se ha desarrollado investigación que ha permitido hacer recomendaciones confiables de fertilización. Por su parte, en papa criolla, se requiere más investigación, especialmente en lo relacionado a la fertilización con nutrientes mayores, secundarios y menores en las variedades nuevas seleccionadas, básicamente no solo por su rendimiento, sino también por el tiempo de brotación de los tubérculos, lo que origina pérdidas en su calidad comercial e industrial y el tubérculo se consume brotado (FEDEPAPA, 2005; Zapata et al. 2006).

Como nutrimento esencial para las planta, en el suelo el calcio (Ca) existe en muchas formas, pero para fines de nutrición, se considera el $\mathrm{Ca}$ en la solución del suelo, el Ca intercambiable y el Ca activo, el cual está constituido por particular finas de carbonato soluble en agua cargada de $\mathrm{CO}_{2}$, que por ionización el calcio pasa a la forma intercambiable (Espinosa, 2003; Lora, 2001). El Ca se absorbe y trasporta en forma iónica, es decir, como catión Ca. Su movilidad es mayor en el apoplasto que en el simplasto de modo que en aquellos órganos que reciben la mayor parte del calcio por el floema es frecuente que reciban un suministro inadecuado del nutrimento (Epstein E Bloom, 2005). El rol del calcio en el ciclo de vida de la planta ha sido bien establecido y revisado extensamente por varios autores (White E Broadley; Hirsechi; Marschemer; citados por Busse $E$ Palta, 2006). Algunas de estas funciones son las siguientes: la mayor parte del calcio, se localiza extracelularmente donde afecta procesos básicos en la pared celular y en las membranas. El pectato de calcio es un componente de la pared celular a la que estabiliza y hace resistente a la degradación por pigalactunorasas. El crecimiento de la raíz depende de $\mathrm{Ca}$ extracelular, que afecta fundamentalmente al alargamiento celular. Pequeñas cantidades de $\mathrm{Ca}$ son necesarias para la mitosis, habiéndose sugerido que las nucleoproteinas se mantienen unidas entre sí mediante puentes cálcicos; también es activador de ciertas enzimas, como la fosfolipasa, la quinasa, la trifosfatasa de la adenosina y otras. Interviene en la circulación de los azúcares, en el metabolismo del nitrógeno (reducción de nitratos), en la neutralización de los ácidos orgánicos y en la reducción de la permeabilidad celular.

Igualmente juega papel importante en la selectividad de la absorción iónica. Por su parte, la ausencia de una cantidad adecuada de $\mathrm{Ca}$ en los tubérculos ha sido asociada con una alta incidencia de defectos fisiológicos, tales como corazón hueco, centro marrón y manchas internas marrones (Björn et al. 2006; Ozgen et al. 2006). Trabajos empleando el radioisótopo ${ }^{45} \mathrm{Ca}$ indicaron que colocando el fertilizante en el tubérculo y en el área estolonífera durante la época de crecimiento mostraron un incremento del contenido del calcio dentro del tubérculo y que el elemento es transportado al tubérculo vía xilema con el agua y las raíces en el estolón asociado con el suministro de agua y calcio al tubérculo en desarrollo. Por otra parte, la demanda transpiracional es determinante en la distribución del calcio dentro de la planta (Busse E Palta, 2006; Larcher, 2003). Además, una adecuada relación del calcio con otros elementos, como potasio, magnesio y azufre es importante dentro de la planta (Malavolta, 1994; Bello E Blanco, 2001).

Con relación a los fertilizantes portadores de Ca son varios los materiales para corregir deficiencias de este nutrimento. En general, las enmiendas para corregir la acidez del suelo, tales como los carbonatos y los 
óxidos e hidróxidos de calcio pueden aportar cantidades elevadas del elemento y, por un aumento del calcio intercambiable, suministrar a la planta el nutrimento. Además, el sulfato de calcio puede ser una fuente de azufre y calcio; igualmente fuentes altamente solubles en agua, tales como el cloruro y el nitrato de calcio son altamente ventajosos, aplicando el fertilizante en solución (Björn et al. 2006; Espinosa, 2003).

El boro (B) es uno de los ocho micronutrientes esenciales para el crecimiento y el desarrollo de las plantas. En el suelo, se encuentra bajo cuatro formas (Alarcón, 2002; Golberg, 1997):

-. Formando parte de minerales silicatados de muy baja disponibilidad para las plantas.

-. Presente en la solución y del suelo como ácido bórico no disociado o como anión borato, $\mathrm{B}(\mathrm{OH})_{4}^{-}$.

-. Adsorbido por las arcillas e hidróxidos de hierro y aluminio a valores de $\mathrm{pH}$, especialmente aleolinos.

-. Ligado a la materia orgánica de la que es liberado progresivamente durante el proceso de mineralización o ésta por acción microbiana, constituyéndose así como la principal fuente de los suelos a las plantas.

En general, suelos con bajo contenido de materia orgánica o baja tasa de mineralización, pueden presentar deficiencias de B. La disponibilidad del micronutriente es afectada tanto por los factores que favorecen su fijación como por aquellos relacionados con el clima, material parental, interacciones con otros elementos, materia orgánica y textura del suelo. El pH es uno de los factores que más influye en la disponibilidad del boro para las plantas, siendo mínima con un $\mathrm{pH}$ inferior a cinco $\mathrm{y}$ máximo entre 5 y 7 y 9 a 10 . El encalado disminuye la disponibilidad por fijación sobre los hidróxidos de hierro y de aluminio recién formados. Las arcillas con estructura micácea, como la vermiculita, tienen mayor habilidad de fijación que las esmectitas. Por su parte, arcilla amorfas o no cristalinas, como el alofano y la imogolita presentes en suelos derivados de cenizas volcánicas, tienen elevada capacidad de fijación de aniones, como boratos, fosfatos y molibdatos (Rojas, 1988).

El B es absorbido por las plantas principalmente como ácido bórico no disociado o como anión borato $\mathrm{B}(\mathrm{OH})_{4}^{-}$. Su transporte a la parte aérea es por flujo de transpiración a través del xilema (Marschner, 2003). El nutrimento es móvil en el floema en aquellas especies vegetales que utilizan polioles como metabolito fotosintético primario (Brown \& Barry, citados por Avila \& Ruiz, 2003), tales como maní, nectarines, almendro, manzano, melocotón, níspero, olivo, pera, uva, ciruela y apio.

Para cultivos, como la papa, en caso de hacer aplicaciones foliares es recomendable efectuar varias que cubran las hojas especialmente hasta la floración para asegurar la síntesis de fotosintetatos, en los cuales el B es necesario. Se anota que en la mayoría de los suelos dedicados al cultivo de papa en Colombia existe deficiencia de B, por lo cual, se hace necesario la aplicación del nutrimento bien sea foliar o edáfica (Barrera, 2000; Lora et al. 2006; Lora et al. 2004). Son varias las funciones propuestas para el boro a saber: transporte de azúcar, síntesis de la pared celular, lignificación, estructura de la pared celular, metabolismo de los carbohidratos, metabolismo del RNA, respiración, ácido indolacético, membranas, metabolismo de fenoles, germinación del polen, crecimiento de tubo polínico y síntesis de proteínas (Marschner, 2003; Epstein E Bloom, 2005). Para la determinación de $\mathrm{B}$ disponible en suelos, se han evaluado varias metodologías, incluyendo los síntomas foliares de deficiencia y de toxicidad (Vargas et al. 2002; Lucho et al. 2005). Los extractantes de B disponibles más empleados son $\mathrm{HCl} 0,05 \mathrm{mg}, \mathrm{Ca}\left(\mathrm{H}_{2} \mathrm{PO}_{4}\right), 0,08 \mathrm{M}$ y agua caliente. Además, la determinación colorimétrica del complejo con azometina-H (Lora, 1998). Es importante tener en cuenta que el límite de boro en el medio de crecimiento entre toxicidad y deficiencia es muy estrecho, por lo cual, este nutrimento debe ser manejado con cuidado (Lora, 2007).

Se debe considerar que el comportamiento de la papa a la aplicación de boro puede depender de la variedad, tal como ha sido soportado en Solanum tuberosum (Abella E Gerenas, 1984). También, se ha encontrado un efecto significativo de la aplicación de $B$ sobre la materia seca del tubérculo en papa criolla (Ávila E Ruiz, 2003).

El objetivo del presente estudio fue evaluar el efecto de aplicaciones edáficas de Ca y B y su interacción en rendimiento y en calidad de tubérculos en papa criolla (Solanum phureja). 


\section{MATERIALES Y MÉTODOS}

El trabajo se llevó a cabo en el municipio de Funza, vereda El Cacique, con una precipitación anual de $800 \mathrm{~mm}$, temperatura de $15^{\circ} \mathrm{C}$, ubicado a $2620 \mathrm{msnm}$, en un suelo derivado de cenizas volcánicas y clasificado taxonómicamente como Typic Dystrandept, medial isomésico. Con base en el análisis del suelo, se aplicó $\mathrm{N}, \mathrm{P}_{2} \mathrm{O}_{5}$ y $\mathrm{K}_{2} \mathrm{O}$ como grado 13-26-6, hierro y cobre.

La fuente de calcio empleada fue nitrato de calcio, $\mathrm{Ca}\left(\mathrm{NO}_{3}\right)_{2}$ con un $24 \%$ de calcio y $17,5 \%$ de nitrógeno, la de boro fue bórax del $10 \%$ de boro. Para nivelar el nitrógeno, se utilizó urea del $46 \%$ de nitrógeno. El diseño experimental empleado fue el de bloques completos al azar en arreglo factorial 4 x 4 con dos factores (B y Ca) y cuatro niveles. Se hizo análisis de varianza y, donde se observó diferencia significativa, se empleó el método de comparaciones múltiples de Duncan al 0,05\%. Cada tratamiento fue repetido tres veces para un total de 16 tratamientos y 48 unidades experimentales constituidas por cuatro surcos de $5 \mathrm{~m}$ de largo y $1 \mathrm{~m}$ entre surcos, para un área de $20 \mathrm{~m}^{2}$. Los tratamientos fueron: 0-0,5-1$1,5 \mathrm{~kg}-\mathrm{B} / \mathrm{h}$ y y $0-18-36-54 \mathrm{~kg}-\mathrm{Ca} / \mathrm{ha}$, tal como aparece en la tabla 1. Por sitio, se colocaron dos semillas de papa variedad yema de huevo a $40 \mathrm{~cm}$ entre cada uno de ellos. Los fertilizantes, se aplicaron al momento de la siembra; se realizó un control de arvenses y fitosanitario al igual que el aporque, el cual estimula la estolonización que producirá los tubérculos, ayuda a la conservación de la humedad y a la protección contra plagas y enfermedades y, evita el verdeamiento de los tubérculos.

Se cosecharon por unidad experimental los dos surcos centrales y se llevó a t/ha. Se tomó una muestra de $2 \mathrm{~kg}$ por parcela para la clasificación por tamaños: extra, primera, segunda y tercera (FEDEPAPA, 2005). En el tamaño primera, se tomó la densidad aparente, el peso fresco, el peso seco y la humedad. Para el análisis económico, se empleó la metodología empleada por Pérez E Riaño (2001).

Tabla 1. Tratamientos empleados.

\begin{tabular}{|c|c|c|}
\hline $\begin{array}{c}\text { TRATAMIENTO } \\
\text { No. }\end{array}$ & B kg/ha & Ca $\mathbf{k g} / \mathbf{h a}$ \\
\hline T1 & 0 & 0 \\
\hline T2 & 0 & 18 \\
\hline T3 & 0 & 36 \\
\hline T4 & 0 & 54 \\
\hline T5 & 0,5 & 0 \\
\hline T6 & 0,5 & 18 \\
\hline T7 & 0,5 & 36 \\
\hline T8 & 0,5 & 54 \\
\hline T9 & 1 & 0 \\
\hline T10 & 1 & 18 \\
\hline T11 & 1 & 36 \\
\hline T12 & 1 & 54 \\
\hline T13 & 1,5 & 0 \\
\hline T14 & 1,5 & 18 \\
\hline T15 & 1,5 & 36 \\
\hline T16 & 1,5 & 54 \\
\hline & & \\
\hline
\end{tabular}




\section{RESULTADOS Y DISCUSIÓN}

Rendimiento total: Los datos de la tabla 2 muestran efecto significativo de los tratamientos sobre la producción total de tubérculos. De acuerdo al coeficiente de determinación $\mathrm{R}^{2}$, el $80 \%$ de la respuesta encontrada es debido a las aplicaciones de calcio y de boro. Por su parte, el coeficiente de variación de $12,45 \%$ está indicando una buena homogeneidad en las unidades experimentales. Las más bajas producciones, se presentaron con las interacciones $\mathrm{Ca}: \mathrm{B}$, de acuerdo a los tratamientos 12 y 7 . Igualmente hubo efecto negativo con relación al testigo en los tratamientos 6-8-16-1412-7, sugiriendo la no efectividad de la combinación de los dos nutrimentos bajo estudio. Posiblemente, el contenido de $0,29 \mathrm{mg} / \mathrm{kg}$ de $\mathrm{B}$ disponible, considerado medio a bajo, junto con el derivado de la mineralización de la materia orgánica del suelo es un nivel elevado para papa criolla. Es importante, por consiguiente, determinar los niveles críticos de boro en papa criolla en suelo y foliar en varias condiciones agroecológicas y con diferentes variedades, tal como lo reporta Lora (2007), para evitar inducir toxicidad del nutrimento, cuyas consecuencias para la planta pueden ser más graves que una deficiencia. En la tabla 3, por medio de la prueba de comparaciones múltiples de Duncan de los tratamientos con el testigo, se observa que éste fue estadísticamente superior a los tratamientos 14-12-7, señalando un efecto negativo de la aplicación de boro con las dosis de calcio, posiblemente por exceso de boro en el suelo, el cual indujo toxicidad para la planta. El Boro con el Ca forma el tetraborato de calcio, moderadamente soluble en agua caliente y soluble en ácidos diluidos. Los tratamientos 10-4-2 fueron significativamente superiores al testigo, mostrando que la mayor respuesta fue al Ca y que es importante determinar la relación Ca:B adecuada en el suelo y en la planta baja diferentes ambientes agroecológicos.

Tabla 2. Efecto de los tratamientos sobre el rendimientos total de tubérculos.

\begin{tabular}{|c|c|}
\hline $\begin{array}{l}\text { TRATAMIENTOS } \\
\text { (kg/ha) B y Ca. }\end{array}$ & $\begin{array}{c}\text { PRODUCCIÓN * } \\
\text { t/ha }\end{array}$ \\
\hline T4 (0-54) & $30,2 a$ \\
\hline T2 (0-18) & $29,7 a b$ \\
\hline T10 (1-18) & $29,5 a b$ \\
\hline $\mathrm{T} 13(1,5-0)$ & $26,5 a b c$ \\
\hline $\mathrm{T} 11(1-36)$ & $25,3 a b c d$ \\
\hline T9 (1-0) & $24,8 a b c d$ \\
\hline T3 (0-36) & $24,3 \mathrm{bcd}$ \\
\hline T5 $(0,5-0)$ & $24,0 \mathrm{~cd}$ \\
\hline T15 (1,5-36) & $24,0 \mathrm{~cd}$ \\
\hline T1 (0-0) & $23,8 \mathrm{~cd}$ \\
\hline T6 $(0,5-18)$ & $23,5 \mathrm{~cd}$ \\
\hline T8 $(0,5-54)$ & 22,0 cde \\
\hline T16 (1,5-54) & $20,3 \mathrm{de}$ \\
\hline T14 (1,5-18) & $17,7 \mathrm{ef}$ \\
\hline T12 (1-54) & $16,8 f$ \\
\hline $\mathrm{T} 7(0,5-36)$ & $12,8 f$ \\
\hline
\end{tabular}

$R^{2}=0,8013 ; C \cdot V \cdot=12,45$

* Cifras con las mismas letras no difieren estadísticamente, (Duncan 0,05). 
Tabla 3. Prueba de comparaciones mútiples de Duncan con el tratamiento testigo.

\begin{tabular}{|c|c|c|c|}
\hline Tatamientos & Duncan $0,5 \% \mathrm{t} / \mathrm{ha}$ & $\begin{array}{l}\text { Diferencia de } \\
\text { rendimientos t/ha }\end{array}$ & $\begin{array}{c}\text { Diferencia } \\
\text { significativa }\end{array}$ \\
\hline $\mathrm{T} 16-\mathrm{T} 1$ & 5,81 & 3,50 & NO \\
\hline $\mathrm{T} 15-\mathrm{T} 1$ & 5,80 & 0,20 & NO \\
\hline T14 - T1 & 5,79 & 6,10 & $\mathrm{SI}^{* *}$ \\
\hline $\mathrm{T} 13-\mathrm{T} 1$ & 5,77 & 2,70 & NO \\
\hline $\mathrm{T} 12-\mathrm{T} 1$ & 5,76 & 7,70 & $\left.S\right|^{* *}$ \\
\hline $\mathrm{T} 11-\mathrm{T} 1$ & 5,75 & 1,50 & NO \\
\hline $\mathrm{T} 10-\mathrm{T} 1$ & 5,70 & 5,71 & $\left.S\right|^{* *}$ \\
\hline T9 - T1 & 5,70 & 1,10 & NO \\
\hline $\mathrm{T} 8-\mathrm{T} 1$ & 5,66 & 1,80 & NO \\
\hline $\mathrm{T} 7-\mathrm{T} 1$ & 5,62 & 11,00 & $\left.S\right|^{* *}$ \\
\hline $\mathrm{T} 6-\mathrm{T} 1$ & 5,56 & 0,30 & NO \\
\hline $\mathrm{T} 5-\mathrm{T} 1$ & 5,49 & 0,20 & NO \\
\hline $\mathrm{T} 4-\mathrm{T} 1$ & 5,27 & 6,40 & $\left.S\right|^{* *}$ \\
\hline $\mathrm{T} 3-\mathrm{T} 1$ & 5,14 & 0,50 & NO \\
\hline $\mathrm{T} 2-\mathrm{T} 1$ & 4,88 & 5,90 & $\mathrm{SI}^{* *}$ \\
\hline
\end{tabular}

NO: no hay diferencia significativa

${ }^{* *}$ diferencia altamente significativa.

Rendimiento por tamaño del tubérculo: En la tabla 4, se puede observar la producción promedio de cada tratamiento por categorías y su participación porcentual, bajo las aplicaciones edáficas de calcio y boro. Aun cuando estadísticamente no hubo efecto significativo es importante considerar que la menor producción se presentó en la calidad tercera, la cual tiene baja aceptación en el mercado nacional.

Por su parte, la calidad primera se presentó en la mayor proporción en los tratamientos, seguido por las calidades segunda, extra y tercera. Posiblemente, si se desea obtener mayor participación de los tamaños primera y extra, se debe investigar en varias condiciones agroecológicas y con diferentes variedades de papa criolla (Zapata et al. 2006).

Efectos combinados de la aplicación de Ca y B sobre la densidad y la humedad del tamaño primera: La densidad y humedad de los tubérculos son características importantes, pues están articuladas al contenido de almidón y materia seca. En la tabla 4, aparece la densidad y la humedad de los tubérculos tamaño primera que fue el de mayor representación en el rendimiento total. El análisis de tendencia central para densidad indica que no hubo efecto de los tratamientos, ya que el coeficiente de variación fue solo de 3,68\% (Solannas et al. 2005).

Las menores densidades, se presentan en los tratamientos 1-2-3-4, donde la dosis de boro es cero y los niveles de calcio se van incrementando. Por su parte, a medida que la dosis de calcio aumenta en presencia de $0,5 \mathrm{~kg}-\mathrm{B} / \mathrm{ha}$, la densidad aumenta hasta un valor de 1,188, mostrando un efecto positivo de esta dosis de boro en presencia de las dosis variables de calcio.

Es muy posible que la densidad de los tubérculos esté muy ligada a la variedad que se utilice. No se presentaron diferencias en el contenido de humedad de los tubérculos, como lo indica el coeficiente de variabilidad de solo $6,70 \%$. En general es conveniente que la humedad sea baja para que la materia seca sea alta y, por tanto, mayor el peso del tubérculo (Epstein E Bloom, 2005). Posiblemente, como lo consideran también Abella $\mathcal{E}$ 
Tabla 4. Efecto de los tratamientos en la producción y en la calidad de tubérculos.

\begin{tabular}{|c|c|c|c|c|c|c|}
\hline Tratamiento No. & extra t/ha & primera t/ha & segunda t/ha & $\begin{array}{c}\text { tercera } \\
\text { t/ha }\end{array}$ & Densidad & Humedad (\%) \\
\hline T1 & $1,97 a$ & $14,18 a$ & $7,51 a$ & $0,18 a$ & 1.084 & 85 \\
\hline T2 & $4,28 a$ & $16,26 a$ & $8,63 a$ & $0,5 a$ & 1.071 & 83 \\
\hline T3 & $3,51 a$ & $14,61 a$ & $5,97 a$ & $0,24 a$ & 1.093 & 82 \\
\hline T4 & $1,66 a$ & $18,99 a$ & $952 a$ & $0,00 a$ & 1.075 & 83 \\
\hline T5 & $3,77 a$ & $16,24 a$ & $3,98 a$ & $0,00 a$ & 1.088 & 83 \\
\hline T6 & $2,09 a$ & $15,30 a$ & $5,98 a$ & $0,14 a$ & 1.157 & 84 \\
\hline T7 & $1,52 a$ & $8,62 a$ & $2,61 a$ & $0,08 a$ & 1.188 & 84 \\
\hline T8 & $1,69 a$ & $13,88 a$ & $6,38 a$ & $0,04 a$ & 1.188 & 81 \\
\hline T9 & $3,11 a$ & $17,34 a$ & $4,31 a$ & $0,07 a$ & 1.099 & 83 \\
\hline T10 & $5,39 a$ & $17,54 a$ & $6,58 a$ & $0,00 a$ & 1.124 & 84 \\
\hline T11 & $1,19 a$ & $17,67 a$ & $6,21 a$ & $0,22 a$ & 1.094 & 83 \\
\hline T12 & $0.98 a$ & $11,26 a$ & $4,50 a$ & $0,10 a$ & 1.129 & 82 \\
\hline T13 & $2.56 a$ & $17,64 a$ & $5,87 a$ & 0,43 & 1.166 & 83 \\
\hline T14 & $3,52 a$ & $10,22 a$ & $3,77 a$ & $0,15 a$ & 1.089 & 83 \\
\hline T15 & $2,32 a$ & $15,11 a$ & $6,46 a$ & $0,11 a$ & 1.079 & 82 \\
\hline T16 & $2,03 a$ & $13,93 a$ & $4,23 a$ & $0,15 a$ & 1.147 & 82 \\
\hline
\end{tabular}

Cifras con las mismas letras no difieren estadísticamente, (Duncan 0,05).

Densidad y Humedad:

$\begin{array}{llll}\text { Media } & : & 1.117 & : 83 \\ \text { Desviación Standard } & : & 0,041 & : 1.00015 \\ \text { C.V. } & : & 3,68 \% & : 6,70 \%\end{array}$

Gerenas (1984), al igual que la densidad, la humedad puede estar ligada a la variedad y a las condiciones agroecológicas. Estos autores encontraron efecto varietal al realizar una investigación con tres variedades de papa de año (Solanum tuberosum).

Análisis económico: Se consideró el precio de venta de la papa y el valor de los fertilizantes empelados (calcio y boro), según la metodología utilizada por Pérez \& Riaño (2001). Los parámetros utilizados fueron:

- Rendimiento total: sumatoria extra, primera y segunda

- Ingreso total: rendimiento por tamaño y su valor

- Ingreso marginal: valor del rendimiento marginal

- Relación beneficio-costo: ingreso marginal sobre costo variable

- Valor kg extra+primera : : \$340,oo
- Valor kg segunda $\quad$ : $\$ \$ 300,00$

- Valor kg de borax : : \$3.500,o0

- Valor kg de nitrato y calcio : $\quad \$ 1.205,00$

La tabla 5, muestra que los tratamientos con mayor relación beneficio:costo son el T2, donde por cada peso invertido en fertilizando (18kg-Ca/ha) se ganan $\$ 19,23$, continuando con los tratamientos T13 - T10 - T9 - T5 - T4. Por su parte hubo tratamientos donde se presentó pérdida como los T6-T7-T8-T12-T14-T16, por lo cual no son recomendables económicamente. El mayor ingreso marginal, se obtuvo con los tratamientos T4T5-T9-T10-T11-T13 y T-15; posiblemente el T4 es uno de los más atractivos para el agricultor. El importante que el agricultor haga este sencillo análisis económico para que así pueda determinar si la explotación agrícola es o no rentable. 
Tabla 5. Análisis económico.

\begin{tabular}{|c|c|c|c|c|c|}
\hline Tratamiento No. & $\begin{array}{c}\text { Producción total } \\
\text { (t/ha) }\end{array}$ & $\begin{array}{c}\text { Valor total } \\
\text { producción }\end{array}$ & $\begin{array}{c}\text { Total costos } \\
\text { variables }\end{array}$ & $\begin{array}{c}\text { * Ingreso } \\
\text { marginal(\$) }\end{array}$ & $\begin{array}{c}\text { *Relación } \\
\text { Beneficio: } \\
\text { Costo }\end{array}$ \\
\hline T1 & 23,60 & $\$ 7,744,000$ & --- & ---- & 0 \\
\hline T2 & 29,20 & $\$ 9,572,600$ & $\$ 90,375$ & $+\$ 1,828,600$ & $+20,23$ \\
\hline T3 & 24,10 & $\$ 7,951,800$ & $\$ 180,750$ & $+\$ 207,800$ & $+1,15$ \\
\hline T4 & 30,10 & $\$ 9,877,000$ & $\$ 271,125$ & $+\$ 2,133,000$ & $+7,87$ \\
\hline T5 & 24,00 & $\$ 7,997,400$ & $\$ 17,500$ & $+\$ 253,400$ & $+14,48$ \\
\hline T6 & 23,30 & $\$ 7,706,600$ & $\$ 107,875$ & $-\$ 37,400$ & $-0,35$ \\
\hline T7 & 12,70 & $\$ 4,230,600$ & $\$ 198,250$ & $-\$ 3,513,400$ & $-17,72$ \\
\hline T8 & 21,60 & $\$ 7,207,800$ & $\$ 288,625$ & $-\$ 536,200$ & $-1,86$ \\
\hline T9 & 24,70 & $\$ 8,246,000$ & $\$ 35,000$ & $+\$ 502,000$ & $+14,34$ \\
\hline T10 & 29,50 & $\$ 9,770,200$ & $\$ 125,375$ & $+\$ 2,026,200$ & $+16,16$ \\
\hline T11 & 25,00 & $\$ 8,275,400$ & $\$ 215,750$ & $+\$ 531,400$ & 2,46 \\
\hline T12 & 16,70 & $\$ 5,511,600$ & $\$ 306,125$ & $-\$ 2,232,400$ & $-7,29$ \\
\hline T13 & 26,10 & $\$ 8,625,000$ & $\$ 52,500$ & $+\$ 885,000$ & $+16,86$ \\
\hline T14 & 16,50 & $\$ 5,802,600$ & $\$ 142,875$ & $-\$ 1,941,400$ & $-13,59$ \\
\hline T15 & 23,90 & $\$ 7,864,200$ & $\$ 233,250$ & $+\$ 120,200$ & $+0,52$ \\
\hline T16 & 20,10 & $\$ 6,695,400$ & $\$ 323,625$ & $-\$ 1,048,600$ & $-3,24$ \\
\hline
\end{tabular}

- * Ingreso marginal y relación beneficio: positivos

- - Ingreso marginal y relación beneficio: negativos

\section{CONCLUSIONES}

Bajo las condiciones de la presente investigación, se puede concluir:

No hubo efecto de los tratamientos en la densidad y en la humedad de los tubérculos tamaño primera, debido posiblemente a que estos parámetros están ligados a la variedad y a las condiciones agroecológicas, tal como se ha encontrado en S. tuberosum.

En el rendimiento total de los tubérculos hubo efecto significativo de los tratamientos, especialmente con las aplicaciones de calcio.

Aun cuando no hubo efecto de los tratamientos en el tamaño de tubérculos, la mayor producción fue tamaño primera de buena aceptación en el mercado nacional.
Los mayores ingresos marginales, se obtuvieron con las aplicaciones de calcio y éste junto con algunas de las dosis de boro, lo cual es importante desde el punto de vista de la rentabilidad para el agricultor.

\section{BIBLIOGRAFÍA}

ABELLA, J.; GERENAS, E. 1984. Respuesta varietal de la papa (Solanum tuberosusm L.) a la aplicación de boro en un Andept de páramo de Cundinamarca. Trabajo de Grado, Ing. Agrónomo. U.N. Bogotá. 157p.

ALARCÓN, V. 2002. El boro como nutriente esencial. Aspectos fisiológicos y dinámicos en el suelo y planta. Depto. Producción Agraria. Universidad de Cartigana, Chile. 
ÁVILA, F.; RUIZ, R. 2003. Efecto de la aplicación foliar y al suelo del boro sobre la papa criolla en un Andisol de la Calera, Cundinamarca. Trabajo de grado, Ing. Agronómico. U.D.C.A. Bogotá. 114 p.

BARRERA, L. 2000. Boro: Un micronutriente importante en el cultivo de la papa en Cundinamarca y Boyacá. En: Papas Colombianas. I.C.A. p.90-95.

BELLO, J.; G. BLANCO. 2001. Evaluación del sulfato de calcio como fuente de azufre y calcio en papa criolla en un suelo Typic Hapludalfs de Cundinamarca. Tesis Ing. Agr. U.D.C.A. Bogotá. 140p.

BJÖRN, K.; PALTA, J.; CRUMP, P. 2006. Enhancing tuber calcium, concentration may reduce incidence of black spot bruise Injury in potatoes. Hort Science 41(5):1213-1221.

BUSSE, J.; PALTA, J. 2006. Investigating the in vivo calcium transport path to developing potato tuber using $45 \mathrm{Ca}$ : a new concept in potato tuber calcium nutrition. Physiol. Plantarum. 128(2):313-323.

COPETE, L.; GARZON, N. 1992. Eficiencia agronómica de tres fuentes de azufre en cuatro andisoles bajo condiciones de casa de malla. Santafé de Bogotá. U. Nacional, p.4-24.

EPSTEIN, E.; BLOOM, A. 2005. Mineral nutrition of plants, principles and perspectives. Senaver Assoc. Inc. Publ., Sunderland, Massachusetts, U.S.A.

ESPINOSA, J. 2003. Encalado de Suelos Tropicales. En: Manejo Integral de la Fertilidad del Suelo. Soc. Col. Cienc. Suelos, Bogotá D.C. p.75-84.

FEDEPAPA. 2005. Guía para el cultivo de la papa. Bogotá, 229p.

GOLBERG, S. 1997. Reactions of boron with soils. Plant E Soils. 193:35-48.

LARCHER, W. 2003. The utilization of mineral elements. En: Physiological Plant Ecology. $4^{\text {th }}$ edition. Berlin, Alemania. p.185-229.

LORA, R. 1998. Análisis de suelos y material vegetal para micronutrientes. En: Silva Mojica, F. (ed).
Actualidad y futuro de los micronutrientes en la agricultura. Soc. Col. de la Cienc. Suelo. Santafé de Bogotá. p.47-56.

LORA, R. 2001. Los elementos secundarios en el cultivo de papa. Soc. Col. Cienc. Suelo. Bogotá. p.114124

LORA, R. 2007. Contaminación por elementos menores y posibles soluciones. Rev. U.D.C.A Act. E Div. Cient. 10(1):5-20.

LORA, R.; MONTAÑEZ, I.; BERNAL, H.; GÓMEZ, R. 2006. Evaluación de la aplicación de Fe-Ca-Mn-ZnB y Mo en papa criolla en un Pachic Melanudands de Cundinamarca. Suelos Ecuat. 36(1):5-12.

LORA, R., PÁEZ, D.; GUZMÁN, M. 2004. Respuesta de la papa criolla a fuentes a dosis de fósforo en Cundinamarca, Colombia. Suelos Ecuat. 4 1:1-8.

LUCHO, C.; PRIETO F.; DEL ROZO, M. RODRÍGUEZ, R.; PAGGI, H. 2005. Chemical fractionation of boron and heavy metals in soils irrigated with water in Central México. Agric. Ecos. Enviromen. 108:57-71.

MALAVOLTA, E. 1994. Diagnóstico foliar. En: Fertilidad de Suelos, Diagnóstico y Control. Soc. Col. de la Ciencia del Suelos. Santafé de Bogotá. p.57-98.

MARSCHNER, H. 2003. Mineral nutritions of higher plants. $2^{\text {nd }}$ ed. Academic Press, S. Diego, California. 889p.

OZGEN, S.; KARLSSON, B.H.; PALTA, J.P. 2006. Responses of potatoes to supplemental calcium application under field conditions. Am. J. Potatoe Res: 83:195-206.

PÉREZ, Y; RIAÑO. A. 2001. Factibilidad económica y financiera de un cultivo de papa criolla (Solanum phureja Juz et Buk) con aplicaciones de NPK en el Municipio de Cogua, Cundinamarca. Trabajo de grado Ing. Agr. Corporación Universitaria de Ciencias Aplicadas y Ambientales U.D.C.A. Bogotá D.C. $89 p$. 
ROJAS, A. 1988. Mineralogical influences on boron adsorption in soils from Colombia. Thesis $\mathrm{PhD}$ in Soil. U. California, Riverside. 118p.

SOLANNAS, A.; SALAFRANCA, L; FAUQUET J.; NUÑEZ, I.. 2005. Estadística descriptiva de las ciencias del comportamiento. Thomson Ed.. Madrid, España. 619p.
VARGAS, C.; BALLESTEROS, I; TRIANA, P. 2002. Evaluación de extractantes para la determinación de boro disponible en suelos. Suelos Ecuat.32:84-89.

ZAPATA, J.; NAVAS, G.; TAMAYO, A.; DÍAZ, C. 2006. Manejo agronómica de la papa criolla para el procesamiento industrial. CORPOICA, C.I. La Selva, Rionegro, Antioquia. 44p.

Recibido: agosto 6 de 2007

Aceptado: octubre 8 de 2007 